\title{
Comparación del desempeño de un controlador PID sobre el proceso de nivel usando un controlador lógico programable y un sistema embebido
}

\author{
Comparison of the performance of PID controller on level process \\ using programmable logic controller and embedded systems
}

\author{
Leonardo Antonio Bermeo Varon ${ }^{1 *} \quad$ Juan Gonzalo Álvarez ${ }^{2} \quad$ William Mantilla Arenas ${ }^{2}$ \\ Recibido 16 de mayo de 2019, aceptado 7 de septiembre de 2021 \\ Received: May 16, 2019 Accepted: September 7, 2021
}

\begin{abstract}
RESUMEN
Los controladores proporcional-integral-derivado (PID) son usados ampliamente en sistemas de control industrial, así como en el sector académico sobre la teoría del control, debido a su simplicidad funcional y robusto desempeño, principalmente. En este documento se presenta la comparación del desempeño de un controlador PID, implementado con dos tecnologías diferentes, (i) un controlador lógico programable SIEMENS SIMATIC S7-1200 y (ii) un sistema embebido basado en RASPBERRY PI. Los parámetros fueron ajustados con iguales condiciones para ambas tecnologías. El control se realizó sobre una planta de nivel en la plataforma ubicada en la sala de control de procesos y sistemas computarizados del Centro de Electricidad y Automatización Industrial (CEAI), SENA en el Valle del Cauca, Colombia. Los resultados obtenidos indican que, para ambas tecnologías bajo las mismas condiciones, los controladores tienen el mismo desempeño en términos de tiempo de establecimiento, tiempo de subida, tiempo de retardo, tiempo pico (tiempo donde se presenta el sobrepaso máximo) y error de estado estacionario, y se diferencian en el esfuerzo de control donde el PLC realiza 2,10\% menos esfuerzo de control que el sistema embebido y un sobrepaso de señal de 2,05\% en comparación con el 4,32\% del sistema embebido. Estos resultados muestran que la implementación de un controlador con un sistema embebido es una buena alternativa de bajo costo para procesos industriales con similares resultados que un controlador implementado en un PLC.
\end{abstract}

Palabras clave: Controlador PID, RASPBERRY PI, PLC, sistema embebido.

\begin{abstract}
Proportional-integral-derivative (PID) controllers are widely used in industrial control systems and control theory academia, mainly due to their functional simplicity and robust performance. This paper presents the performance comparison of a PID controller, implemented with two different technologies, (i) a SIEMENS SIMATIC S7-1200 programmable logic controller and (ii) an embedded system based on RASPBERRY PI. The parameters were set with the same conditions for both technologies. The control was performed on a level system on the platform located in the process control room and computerized systems at the Electricity and Industrial Automation Center (CEAI), SENA in Valle del Cauca, Colombia. The results obtained indicate that, for both technologies under the same conditions, the controllers have the same performance in terms of settling time, rise time, delay time, peak time (time where the maximum overshoot occurs), and steadystate error. Makeover, they differ in the control effort where the PLC performs $2.10 \%$ less control effort than the embedded system and a signal overshoot of $2.05 \%$ compared to $4.32 \%$ of the embedded system. These results show that controller implementation with an embedded system is an excellent low-cost alternative for industrial processes with similar results as a controller implemented in a PLC.
\end{abstract}

Keywords: PID controller, Raspberry PI, PLC, Embedded Systems.

1 Universidad Santiago de Cali. Facultad de Ingeniería. Cali, Colombia. E-mail: leonardo.bermeo00@usc.edu.co

2 Centro de Electricidad y Automatización Industrial del SENA. Regional Valle del Cauca, Cali, Colombia.

E-mail: jgalvarez@sena.edu.co; wmantilla@sena.edu.co

* Autor de correspondencia: leonardo.bermeo00@usc.edu.co 


\section{INTRODUCCIÓN}

El controlador Proporcional-Integral-Derivativo (PID) es un tipo de control con realimentación, que utiliza una función para describir el comportamiento de la salida de un sistema con respecto a su entrada de referencia. Su propósito fundamental es hacer que el error en estado estacionario sea cero. Este controlador está caracterizado por tres parámetros: (i) el proporcional que depende de la información actual del sistema, (ii) el integral que depende de la información pasada del sistema, el cual establece que el error de estado estacionario del sistema sea cero de manera asintótica en el tiempo y (iii) el derivativo que realiza un efecto predictivo del sistema.

Un controlador PID es una estrategia suficiente para solucionar problemas de control de muchas variables industriales, como la temperatura, el flujo, la presión y el nivel. La dinámica de estas variables puede ser descrita por sistemas de primer o segundo orden, dependiendo de la aplicación en la que estén presentes. Hay que tener en cuenta que en muchos casos este controlador tiene limitaciones en su desempeño, particularmente en especificaciones como el error de estado estacionario, rápida respuesta a cambios en presencia de disturbios y/o cambios en su señal de referencia. A pesar de estas limitaciones, su robustez permite que sea utilizado en la industria, por encima de otros controladores, por ejemplo, los difusos.

Actualmente, existen diversas estrategias de control, las cuales dependiendo de su aplicación y estudio de desempeño, han determinado la mejor estrategia a implementar en un proceso [1-4]. A pesar de existir diferentes tipos de control, en la industria el 95\% de los lazos de control utilizados son de tipo PID [5] y en su mayoría son implementados sobre un Controlador Lógico Programable (PLC) [6-7], los cuales se han convertido en un dispositivo esencial en el momento de implementar un controlador PID [7-8]. Un PLC no solo proporciona el control de un sistema, también puede realizar funciones básicas de control on/off, temporización, secuenciación, aritmética y manejo de datos [9], sistemas de control de movimiento sofisticados, control de procesos, sistemas de control distribuido y redes complejas [10] y sistemas de control difuso [11], garantizando fiabilidad, flexibilidad, capacidad de comunicación, tiempo de respuesta rápida y fácil programación para control en aplicaciones industriales [12], por estas razones su uso en la industria para implementar sistemas de control es extenso.

Por otro lado, los sistemas embebidos actuales han permitido el desarrollo de controladores PID [13-18], los cuales pueden llegar a tener las mismas características de una implementación de un controlador sobre un PLC, especialmente en la flexibilidad de su programación, la capacidad de realizar ajustes de parámetros de forma automática, cambios del modo de control y la posibilidad de implementar control robusto y óptimo. Al igual que un PLC un sistema embebido también permite realizar diferentes funciones a las de un controlador, como por ejemplo procesamiento de datos [19]. Una característica, no menos importante, de un sistema embebido es su bajo costo con respecto a un PLC, lo que es importante en la optimización de recursos de la industria.

Teniendo en cuenta las características de las tecnologías descritas, en este artículo se presenta la comparación de los índices de desempeño de un controlador PID implementado en un PLC SIEMENS S7-1200 y en un sistema embebido basado en RASPBERRY PI. Los controladores son evaluados bajo las mismas condiciones de diseño sobre un sistema de nivel de la plataforma de formación en control de procesos y sistemas computarizados del Centro de Electricidad y Automatización Industrial (CEAI), del SENA regional Valle del Cauca, Colombia.

\section{CONTROLADOR PID}

Un controlador PID es un control con realimentación en lazo cerrado ampliamente utilizado en los sistemas de control industrial [5]. Un controlador PID corrige el error entre la medición de la variable del proceso y una referencia deseada, calculando y ejerciendo una acción correctiva, denominada acción de control, que ajusta el proceso hasta que el error sea cero. El cálculo del controlador PID involucra tres parámetros por separado. El valor proporcional que determina la acción al error actual, la integral que determina la acción con base en la suma de los errores pasados y la derivada que determina la acción anticipada a los cambios en su salida [20-21]. Las tres constantes son sintonizadas para que el PID proporcione una acción de control que satisfaga los requisitos específicos del proceso. La respuesta 
del controlador se puede describir en términos de la capacidad de respuesta de la acción de control a un error, al grado en que el controlador sobrepasa el punto de referencia y al grado de oscilación del sistema, como se define en la ecuación (1).

$$
u(t)=K_{p}\left[e(t)+\frac{1}{T_{i}} e \int_{0}^{t} e(t) d t+T_{d} \frac{d}{d t} e(t)\right]
$$

donde $u(t)$ es la acción de control, $e(t)$ es el error, $K_{p}$ es el parámetro proporcional, $T_{i}$ es el parámetro integral y $T_{d}$ es el parámetro derivativo. $K_{p}, T_{i}$ y $T_{d}$ son los parámetros ajustables para sintonizar el comportamiento del lazo de control. Esta función en el dominio de la frecuencia está definida en la ecuación (2).

$$
\frac{U(s)}{E(s)}=K_{p}\left(1+\frac{1}{T_{i} s}+T_{d} s\right)
$$

En las Figura 1 y Figura 2 se presentan un esquema y un diagrama de bloques de un controlador PID, respectivamente, donde se observan los parámetros que intervienen en el controlador [6].

\section{IDENTIFICACIÓN DEL SISTEMA E IMPLEMENTACIÓN DEL CONTROLADOR PID}

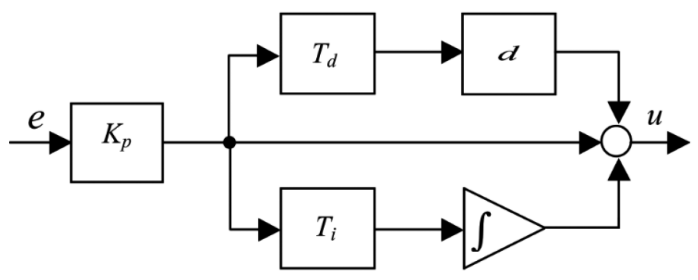

Figura 1. Diagrama esquemático de un controlador PID.

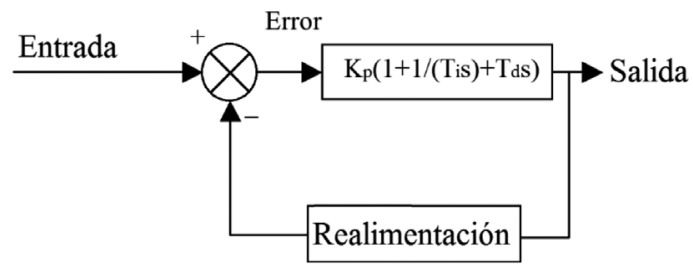

Figura 2. Diagrama de bloques de un controlador PID.
El Servicio Nacional de Aprendizaje (SENA), dentro de su proceso de formación en el área de control de procesos y sistemas computarizados, cuenta con una plataforma técnica compuesta por una planta multiprocesos con dispositivos de medición, transmisores de nivel, flujo y temperatura, tecnología HART, válvulas de control servo actuadas, bombas centrífugas, compresor, intercambiador de calor y un sistema de calefacción a través de resistencias eléctricas. En esta planta se realizan estrategias de control de lazo abierto, lazo cerrado y control en cascada, además cuenta con acceso remoto a través de internet y un sistema de monitoreo de la planta a través de una cámara Web. Para el control de procesos dispone de cuatro (4) tipos de controladores: (i) controlador Allen Bradley, de la serie CompactLogix L24ER, (ii) un controlador SIEMENS SIMATIC S71200, (iii) Controlador SIEMENS SIMATIC S5-1500 y (iv) un sistema embebido basado en RASPBERRY PI [22]. En la Figura 3 se presenta Planta didáctica para Formación Remota en Control de Procesos. El proceso de identificación del sistema, sintonización de los parámetros del PID e implementación de los controladores PID sobre el PLC y el sistema embebido, es realizado sobre la planta de nivel de la plataforma de procesos industriales del Centro de Electricidad y Automatización Industrial CEAI del SENA regional Valle del Cauca, Colombia. En la Figura 4 se presenta el esquema P\&ID de una sección de la plataforma, particularmente el sistema de nivel a controlar, donde TK 102 es el tanque, LCV 102 es la válvula de salida del tanque, LCV 100 es la válvula de llenado del tanque, LT 101 es el transmisor de nivel, FT 100 es el transmisor de flujo, TT101 es el transmisor de temperatura y CP 100 es el panel de control.

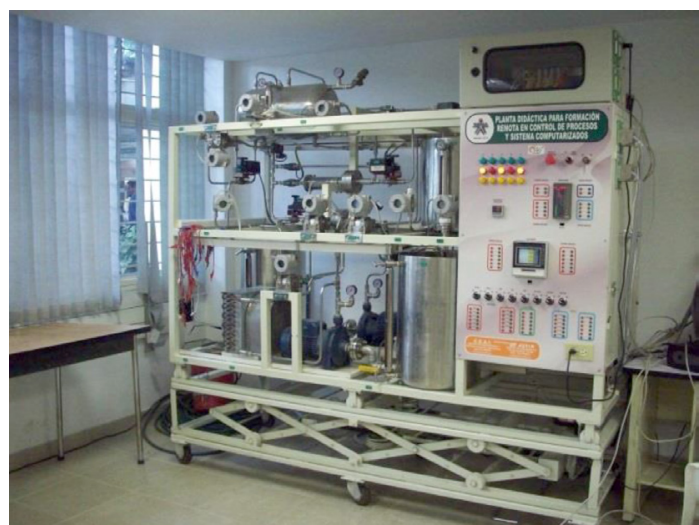

Figura 3. Sistema para formación remota de control de procesos. 


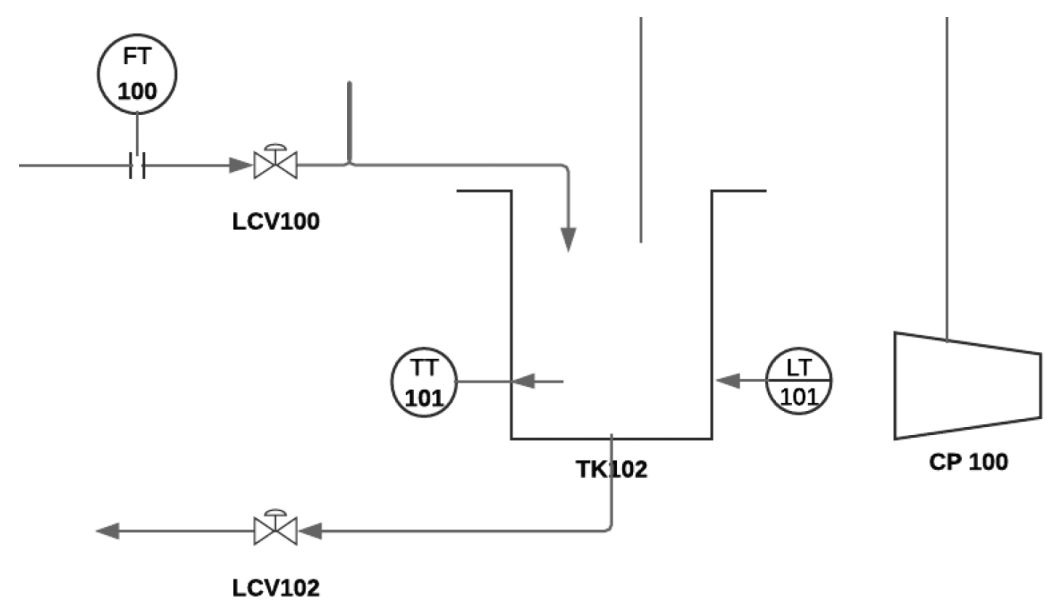

Figura 4. Diagrama P\&ID parcial de la planta didáctica para formación remota en control de procesos (Sistema de nivel).
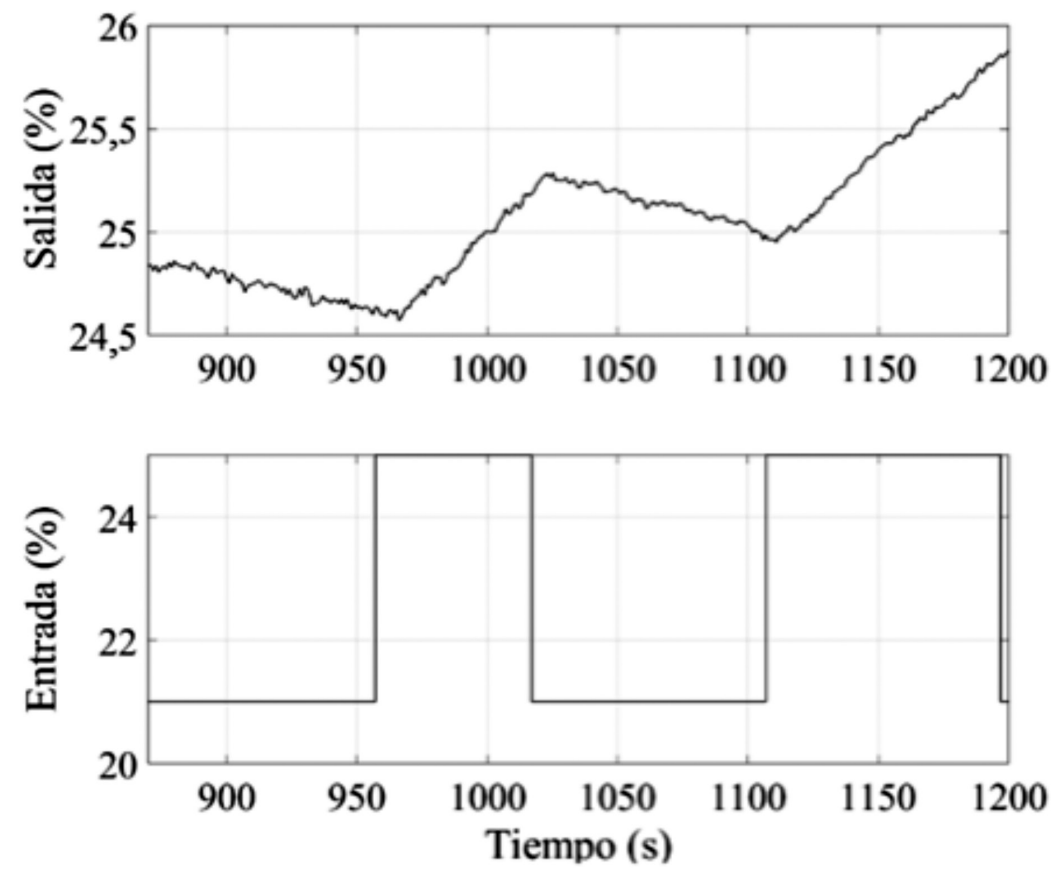

Figura 5. Salida y entrada de la planta de nivel en lazo abierto.

Identificación del Sistema y Sintonización de los Parámetros del PID

El proceso de identificación y sintonización de los parámetros del PID consistió en el registro del comportamiento de la salida del sistema de nivel en lazo abierto ante variaciones de tipo escalón aleatorio en su entrada, esto es, entre el $21-25 \%$ de apertura de la válvula (LCV100) durante $3600 \mathrm{~s}$, en la Figura 5, se registra un segmento de la entrada y salida del proceso en lazo abierto.

Las señales de entrada y salida obtenidas en la planta de nivel en lazo abierto son procesadas en la aplicación "ident” de Matlab MathWorks®, la cual permite identificar o estimar un modelo matemático y/o función de transferencia de una planta sin 
necesidad de conocer los parámetros técnicos de la planta o de conocerla realmente. El proceso permitió, a través de datos reales del sistema encontrar un modelo con un grado de correlación alto.

Se realizaron tres estimaciones sobre los datos buscando obtener el modelo que más porcentaje de correlación proporcione respecto a una señal de validación diferente a los datos utilizados en la identificación. En la Figura 6 se presentan diferentes modelos obtenidos de donde se escogió el modelo que mejor estime el comportamiento del sistema. Como se puede observar el modelo (M3) es el que tiene mejor comportamiento con un $90,18 \%$ de exactitud sobre los datos del sistema, la ecuación (3) presenta el sistema. Una vez obtenido el modelo se hace uso de la herramienta "PidTuner" de Matlab MathWorks® para establecer los parámetros de sintonía que cumplan los siguientes requerimientos: (i) sobre impulso por debajo del 10\%, (ii) tiempo de establecimiento alrededor de 6 minutos y (iii) error de estado estacionario cero. Los parámetros de control, desempeño y robustez obtenidos después de la sintonización se presentan en la Tabla 1, donde observamos que $K_{p}=5,4884, T_{i}=161,5336$ y $T_{d}=0,0000$.

$$
G(s)=\frac{1,14}{(286,45 s+1)(9,93 s+1)}
$$

\section{Sistema de control con el PLC SIEMENS S7-1200} El PLC Siemens S7-1200 consiste en un controlador con interfaz PROFINET integrada

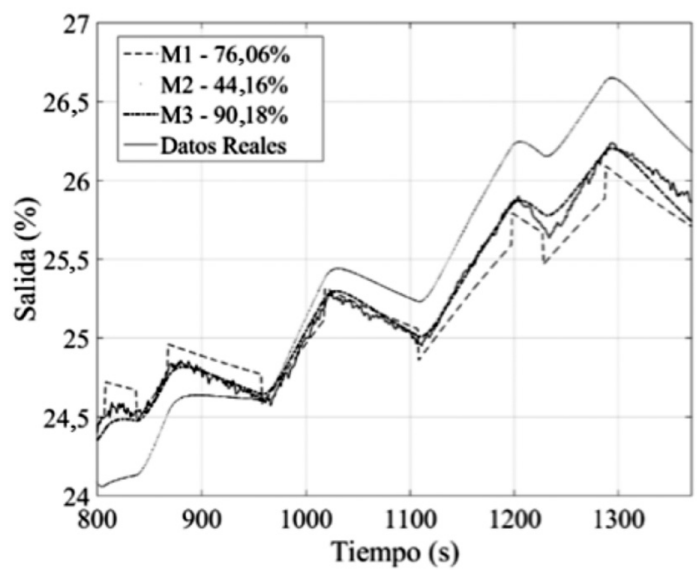

Figura 6. Salida del sistema y modelos estimados (M) por la función "ident". para comunicaciones, con dispositivos HMI y otros controladores SIMATIC, posee funciones de control de movimiento, control en lazo cerrado, contadores, salidas y entradas análogas y digitales, módulos de expansión de entradas/salidas e interfaz de comunicaciones [23].

El algoritmo PID del PLC está definido por la ecuación (4), la cual no requiere que los parámetros sean definidos manualmente, ya que durante el proceso de control estos parámetros se adaptan al sistema [21]. Sin embargo, para esta experiencia los parámetros se definieron manualmente utilizando los parámetros obtenidos en el proceso de sintonización del PID descritos en la Tabla 1, note que el parámetro derivativo $T_{d}=0,000$, lo que indica que el controlador carece de acción derivativa.

$y=K_{p}\left[(b \cdot w-x)+\frac{1}{T_{i} s}(w-x)+\frac{T_{d} s}{a \cdot T_{d} \cdot s+1}(c \cdot w-x)\right](4)$

En la ecuación (4), $b$ es la ponderación de la acción de control proporcional, $w$ es la referencia, $x$ es el valor real, $a$ es el coeficiente para el retardo de la acción derivada y $c$ es la ponderación de la acción derivativa. En la Figura 7 se presenta el diagrama de bloques del algoritmo de PID en el PLC SIEMENS SIMATIC S7-1200 [24].

\section{Sistema de control con Raspberry PI (sistema embebido)}

Raspberry PI es un controlador de placa única, reducida y simple, de bajo de costo desarrollado

Tabla 1. Sintonización de parámetros para El PID.

\begin{tabular}{|l|c|}
\hline \multicolumn{2}{|c|}{ Parámetros de Control } \\
\hline $\mathrm{K}_{\mathrm{p}}$ & 5,4884 \\
\hline $\mathrm{T}_{\mathrm{i}}$ & 161,5336 \\
\hline $\mathrm{T}_{\mathrm{d}}$ Desempeño y Robustez \\
\hline \multicolumn{2}{|c|}{0,000} \\
\hline Tiempo de subida & $64,7 \mathrm{~s}$ \\
\hline Tiempo de estabilidad & $370 \mathrm{~s}$ \\
\hline sobrepaso & $6,4 \%$ \\
\hline Pico & 1,06 \\
\hline Margen de Ganancia & $\infty \mathrm{dB} @ \infty \mathrm{rad} / \mathrm{s}$ \\
\hline Margen de fase & $71^{\circ} \mathrm{C} @ 0,0219 \mathrm{rad} / \mathrm{s}$ \\
\hline Estabilidad en lazo cerrado & Estable \\
\hline
\end{tabular}




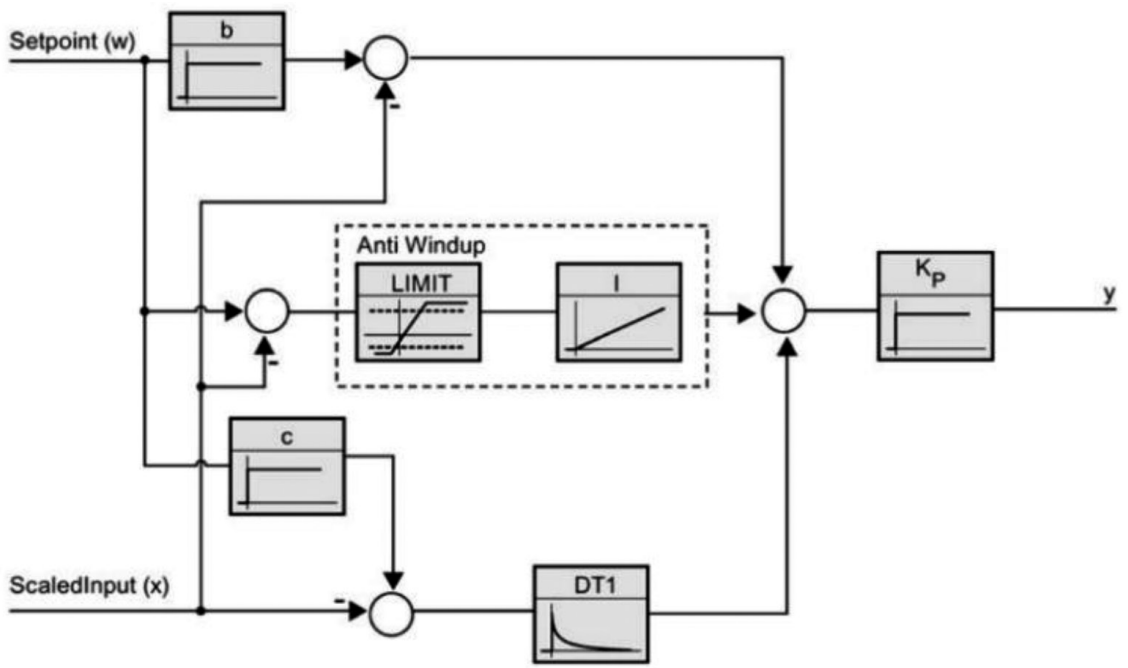

Figura 7. Esquema de control del PID del PLC SIEMENS SIMATIC S7-1200.

en Reino Unido por la Fundación Raspberry PI, el sistema está constituido por un software de fuente abierta con sistema operativo Raspbian adaptado de Debian, permite usar otros sistemas operativos, incluido una versión de Windows ${ }^{\circledR} 10$. Incluye un procesador Broadcom, memoria RAM, GPU, puertos USB, HDMI, Ethernet, 40 pines GPIO y un conector para cámara.

La Raspberry PI, al ser un sistema embebido, requiere que su programación sea de forma discreta. En ese sentido se considera el algoritmo de control PID definido en el tiempo para el PLC SIEMENS S7-1200 [24] de forma discreta, asegurando de esta manera las mismas condiciones del controlador PID implementado en el PLC. En la ecuación (4) se presenta el controlador, a esta ecuación se le realiza la transformada $z$ y es expresado en términos de ecuaciones de diferencia. En la ecuación (5) se presenta la transformación bilineal por el método de Tustin [25], que define al operador " $s$ " para realizar la transformación matemática del controlador. Las ecuaciones (6-8) representan la transformada $z$ del sistema.

$$
s=\frac{2}{T} \frac{1-z^{-1}}{1+z^{-1}}
$$

Sustituyendo la ecuación (5) en la ecuación (4), obtenemos las ecuaciones (6-8) que define el sistema en términos de variable discreta $z$ :

$$
\begin{gathered}
y_{p k}=K_{p}\left(b \cdot w_{k}-x_{k}\right) \\
y_{i k}=\frac{T}{2 T_{i}}\left(w_{k}-x_{k}\right)+\frac{T}{2 T_{i}}\left(w_{k-1}-x_{k-1}\right)+y_{i k-1} \\
y_{d k}=\frac{T-2 a T_{d}}{T+2 a T_{d}} y d k-1+\frac{2 T_{d}}{T+2 a T_{d}}\left(c \cdot w_{k}-x_{k}\right)- \\
\frac{2 T_{d}}{T+2 a T_{d}}\left(c \cdot w_{k-1}-x_{k-1}\right)
\end{gathered}
$$

Donde $y_{p k}$ representa la acción proporcional del controlador, $y_{l k}$ representa la acción integral y $y_{d k}$ representa la acción derivativa. El subíndice $k$ indica la muestra actual, $k$-1 indica la muestra anterior y $T$ indica el tiempo de muestreo. Las ecuaciones (6-8) se suman y proporcionan la salida del controlador. Finalmente, las ecuaciones (6-8) son implementadas en el sistema embebido.

\section{RESULTADOS Y DISCUSIONES}

La comparación entre los controladores PID implementados sobre el PLC SIEMENS SIMATIC S7-1200 y el sistema embebido basado en Raspberry PI, fue realizada sobre la variable de nivel (TK102) del sistema, previamente estable a un $30 \%$, ante una entrada escalón $u(t)$ de $t=$ $1920 \mathrm{~s}$, como se describe en la ecuación (9). El tiempo de muestreo para ambos sistemas es $T_{s}=1 \mathrm{~s}$. 


$$
u(t)=\left\{\begin{array}{c}
0,3(30 \%), 0<t \leq 600 s \\
0,34(34 \%), 600 s<t \leq 1320 s \\
0,3(30 \%), 1320 s<t \leq 1920 s
\end{array}\right.
$$

Para el análisis comparativo de desempeño se analizaron el tiempo de estabilización, sobrepaso, tiempo pico (tiempo donde se presenta el sobrepaso máximo), tiempo de subida (tiempo donde alcanza la referencia por primera vez), tiempo de retardo (tiempo en alcanzar el $50 \%$ de la referencia), error de estado estacionario y esfuerzo de control. Se realizaron 10 pruebas en el sistema para cada controlador en iguales condiciones, esto es, parámetros del PID presentados en la Tabla 1. También, se realiza la comparación de los sistemas ante un disturbio.

En las Figura 8 y Figura 9 se muestra la salida del sistema, el esfuerzo de control y error del sistema realizado en el PLC y el sistema embebido Raspberry

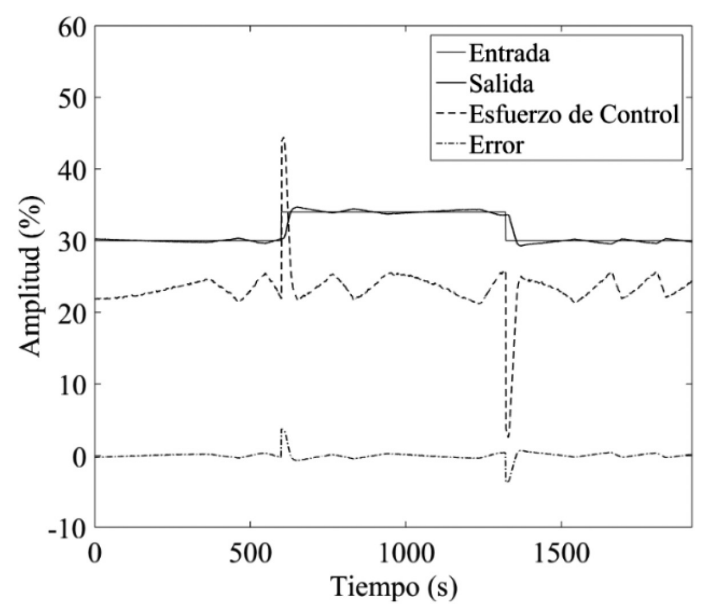

Figura 8. Controlador PID con el PLC SIEMENS S7-1200.

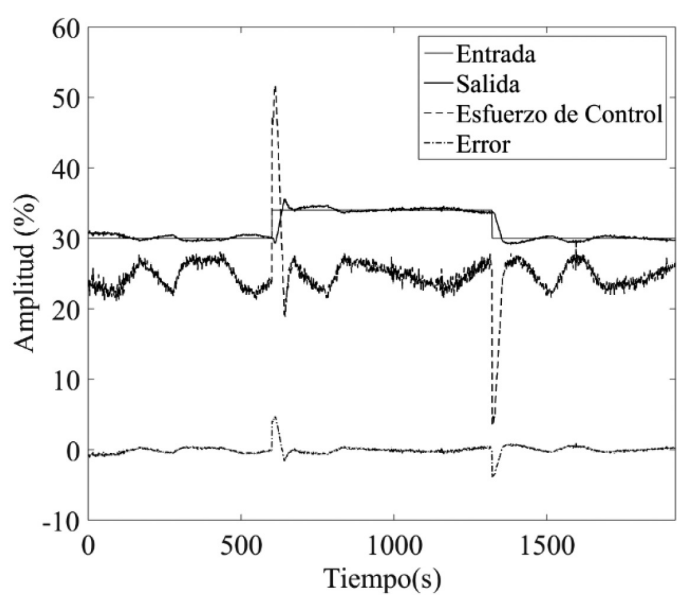

Figura 9. Controlador PID con el sistema embebido.

PI, respectivamente. En la Tabla 2 se muestran los resultados promedio y desviación estándar del tiempo de estabilización, tiempo de subida, sobrepaso máximo, tiempo pico, tiempo de retardo y error en estado estacionario. Estos resultados nos indican que ambos controladores cumplen con las características de desempeño presentadas en la Tabla 1.

Comparando los controladores por medio del promedio y la desviación estándar de las 10 experiencias de cada controlador, se observan los siguientes comportamientos: (i) el controlador implementado con el PLC presenta un sobrepaso y variación menor que el presentado en el sistema embebido, lo que significa que el controlador implementado con el PLC realiza menos esfuerzo de control, a pesar que el tiempo pico donde ocurre el sobrepaso máximo en promedio es mayor y con una dispersión de datos relativamente alta. Sin embargo, ambos controladores están dentro de los parámetros de diseño del $6,4 \%$, (ii) en el tiempo de subida y de

Tabla 2. Parámetros de desempeño del sistema de nivel.

\begin{tabular}{|l|c|c|c|c|}
\cline { 2 - 5 } \multicolumn{1}{c|}{} & \multicolumn{2}{c|}{ PLC } & \multicolumn{2}{c|}{ Sistema Embebido } \\
\cline { 2 - 5 } \multicolumn{1}{c|}{} & Promedio & Desviación estándar & Promedio & Desviación estándar \\
\hline Tiempo de estabilización (s) & 384,70 & 98,56 & 342,83 & 32,80 \\
\hline Tiempo de subida (s) & 30,60 & 1,17 & 32,50 & 1,51 \\
\hline Tiempo de retardo (s) & 19,10 & 0,73 & 22,66 & 1,75 \\
\hline Tiempo pico (s) & 57,10 & 12,75 & 41,66 & 1,96 \\
\hline Porcentaje Sobrepaso Máximo (\%) & 2,05 & 0,22 & 4,32 & 0,41 \\
\hline Error de estado estacionario (RMS) & 0,1711 & 0,0527 & 0,2078 & 0,0301 \\
\hline
\end{tabular}


retardo se observa que los dos controladores tienen un comportamiento similar, esto es, tiempo de retardo de $20 \mathrm{~s}$ y tiempo de subida de $31 \mathrm{~s}$ aproximadamente, (iii) en el tiempo de estabilización se observa que el sistema implementado con el sistema embebido es más rápido que el presentado con el PLC con una diferencia de $40 \mathrm{~s}$ aproximadamente, además con dispersión menor con respecto al PLC de 32,80 s, mientras que el PLC presenta una dispersión de 98,56 s, (iv) en el error de estado estacionario el comportamiento de los controladores es prácticamente similar, 0,17 para el PLC y 0,20 para el sistema embebido. Sin embargo, el PLC presenta una dispersión de datos mayor, esto se ve reflejado en la desviación estándar de 0,0527 , mientras que el sistema embebido es de 0,0301 .

Con el sistema previamente estable en un $30 \%$ del nivel, se realizó la perturbación a los $600 \mathrm{~s}$, la cual consistió en el cierre de la válvula ubicada en la salida del sistema (LCV102) durante $720 \mathrm{~s}$ en un $30 \%$, posteriormente se reestablece la válvula a su estado inicial, esto es, abrirla y suprimir el disturbio. En ambos controladores, cuando la válvula LCV102 se cierra el sistema empieza a compensar el incremento de nivel con el cierre de la válvula LCV100 y en el momento de la apertura de la válvula LCV102 el sistema nuevamente empieza a compensar la caída de nivel con la apertura de la válvula LCV100.

En la Figura 10 se presenta el esfuerzo de control de ambos controladores. Note que cuando el sistema está en reposo, el controlador implementado con

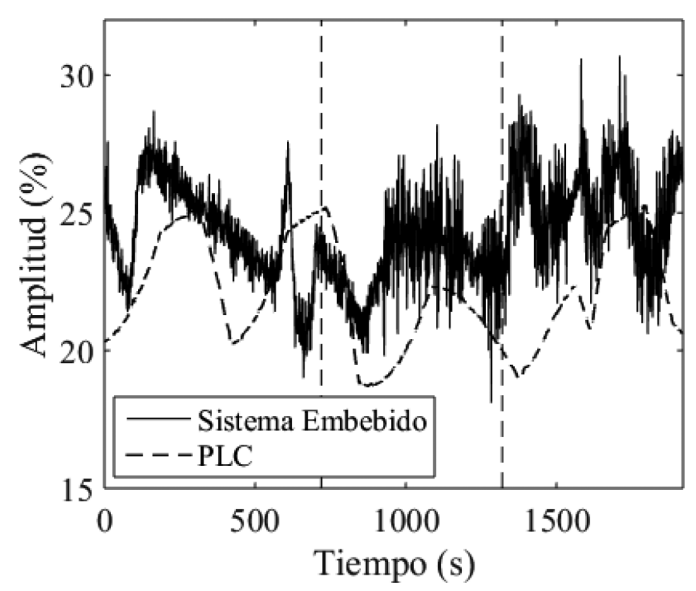

Figura 10. Esfuerzo de control ante un disturbio. el sistema embebido presenta un mayor esfuerzo de control, esto es $24,71 \pm 1,77 \%$ de amplitud, con respecto al $23,07 \pm 1,66 \%$ de amplitud en controlador implementado con el PLC, 1,64\% menos.

Ante la presencia del disturbio, en donde se requiere menos esfuerzo de control, se observa una vez más que el controlador implementado con el sistema embebido presenta mayor esfuerzo de control con un $23,18 \pm 1,44 \%$ de amplitud con respecto a $20,63 \pm 1,22 \%$ en el sistema implementado con el PLC, 2,35\% menos.

En el momento de eliminar el disturbio, el esfuerzo de control en el controlador implementado con el sistema embebido una vez más es mayor con un $25,28 \pm 1,73 \%$ de amplitud con respecto al sistema implementado con el PLC que es de 22,97 $\pm 1,65 \%, 2,31 \%$ menos.

Como podemos observar el controlador implementado en el PLC realiza un menor esfuerzo de control, esto es un $2,10 \%$ en promedio menos que el implementado en el sistema embebido, en coherencia con la respuesta del sistema ante la entrada escalón. Se observa que el esfuerzo de control del sistema embebido es menos estable que el PLC el cual tiene un esfuerzo de control suave, un esfuerzo de control inestable podría afectar el comportamiento de los actuadores con el tiempo. A pesar de este comportamiento, el controlador implementado con el sistema embebido presenta características similares al controlador realizado con el PLC, solo difieren en un $2,10 \%$ en amplitud.

\section{Análisis de Costos de las dos Tecnologías}

En la Tabla 3 se presenta el costo de equipos y accesorios de los dos sistemas de control, incluyendo precios de mantenimiento. Note, que el sistema de control realizado con el sistema embebido, donde el módulo principal es una Raspberry PI, es mucho mas económico tanto de la CPU central como los módulos adicionales para realizar un control PID. En cuanto al mantenimiento también se observa que los costos son menores que con un PLC, a pesar de que el sistema embebido necesita ser atendidos con más frecuencia, esto es, 4 veces por año lo que genera parada en la producción y que dependiendo del sector generaría posibles costos indirectos. Sin embargo, la planeación de mantenimiento es importante para generar el menor impacto posible en la producción. Los costos del sistema basado en le PLC Siemens 
Tabla 3. Parámetros de desempeño del sistema de nivel.

\begin{tabular}{|l|c|c|c|}
\hline \multicolumn{1}{|c|}{ Componente } & & Embebido (USD) & Siemens S7-1214C (USD) \\
\hline Fuente de Voltaje & 1 & 37,14 & 0,00 \\
\hline Sistema de Comunicaciones & 1 & 54,29 & 0,00 \\
\hline CPU Central & 1 & 54,29 & 572,43 \\
\hline Entradas Digitales & 1 & 62,86 & 0,00 \\
\hline Salidas Digitales & 1 & 71,43 & 0,00 \\
\hline Entradas Analógicas & 1 & 80,00 & 339,51 \\
\hline Salidas Analógicas & 1 & 117,14 & 358,86 \\
\hline Software & 1 & 0,00 & 1000,00 \\
\hline Mantenimiento & 1 & $50,00^{*}$ & $120,00^{*} *$ \\
\hline Total (sin mantenimiento) & 1 & 477,14 & 2270,80 \\
\hline Total (con mantenimiento) & 1 & 677,14 & 2510,80 \\
\hline
\end{tabular}

*se requiere 4 mantenimientos por año.

**se requiere 2 mantenimientos por año.

se ven afectados ya que este requiere del software Simatic Step 7 básico que como se puede observar es de 1000 USD, mientras que el sistema basado en Raspberry PI el software Raspbian es libre (open source). Actualmente, ya esta en el mercado un PLC Raspberry Pi para aplicaciones industriales el cual no tiene diferencias con el sistema aquí implementado y estudiado, el sistema incluye fuente de alimentación, múltiple conectividad (Ethernet, RS-485), y entradas/salidas analógicas y digitales, estos sistemas se encuentran desde los 300 USD hasta los 340 USD, aproximadamente, dependiendo de la capacidad de memoria que se requiera.

\section{CONCLUSIONES}

En este artículo se presentó un análisis comparativo del desempeño de un controlador PID implementado con dos tecnologías, PLC y sistema embebido (Raspberry PI), para controlar la variable de nivel de la plataforma de procesos industriales del Centro de Electricidad y Automatización Industrial CEAI del SENA regional Valle del Cauca, Colombia. Los parámetros que se utilizaron fueron tiempo de estabilización, tiempo de subida, sobrepaso máximo, tiempo pico, tiempo de retardo, error en estado estacionario y esfuerzo de control presencia de disturbios. Los resultados estadísticos mostraron que, (i) en cuanto a tiempo de estabilización, tiempo de subida y tiempo de retardo ambos sistemas presentan similares comportamientos, (ii) en el caso de sobrepaso máximo y tiempo en donde se presenta el pico más alto, el controlador implementado con el PLC presenta mayor dispersión y el sobrepaso ocurre más tarde, que en el sistema embebido. Sin embargo, este sobrepaso es menor, lo que indica que el PLC realiza menos esfuerzo de control que el controlador implementado con el sistema embebido, tanto para respuesta ante el escalón como para presencia de disturbios, el tiempo donde se presenta el pico más alto es mayor en el controlador con el PLC, sin embargo esto no afecta el desempeño del sistema, (iii) en cuanto al error de estado estacionario ambos sistemas tienen un buen desempeño, el controlador con el PLC tiene un error menor, (iv) el controlador implementado con el sistema embebido presenta menor variabilidad en el tiempo de estabilización y error de estado estacionario, esto se ve reflejado en la desviación estándar, lo que indica una menor dispersión de resultados entre prueba y prueba y (v) el PLC necesita menos esfuerzo de control para alcanzar la referencia y estabilizar el sistema, esto se ve reflejado en las pruebas realizadas ante un disturbio, en el cual el PLC realiza 2,10\% menos esfuerzo de control que el sistema embebido, sin embargo, esta diferencia no indica que el sistema embebido no sea adecuado debido a que esta diferencia es mínima.

Un sistema embebido es una excelente opción para implementar un controlador industrial realimentado debido principalmente a que su desempeño es similar al controlador implementado con un PLC, con respecto al tiempo de estabilización, tiempo de subida, tiempo de retardo y error de estado 
estacionario, solo difiere en el esfuerzo de control en donde se presenta un 2,10\% mayor, sin perjudicar los parámetros de diseño del sistema.

Finalmente, el sistema basado en Raspberry PI se puede considerar de bajo costo, ya que su implementación es mucho menor que un sistema basado en PLC, esto lo convierte en una excelente alternativa industrial, sobre todo si la compañía es pequeña o está empezando, teniendo en cuenta que se obtienen resultados muy similares en cuanto a su desempeño y robustez.

\section{REFERENCIAS}

[1] L. Angel and J. Viola. "Design and statistical robustness analysis of FOPID, IOPID and SIMC PID controllers applied to a motor-generator". IEEE Latin American Transactions. Vol. 13 N $^{\circ} 12$, pp. 3724-3734. 2015. ISSN: 1548-0992. DOI: 10.1109/ TLA.2015.7404900.

[2] J. Viola and L. Angel. "Factorial design for robustness evaluation of fractional PID controllers". IEEE Latin American Transactions. Vol. $13 \mathrm{~N}^{\circ}$ 5, pp. 1286-1293. 2015. ISSN: 1548-0992. DOI: 10. 1109/ TLA.2015.7111981.

[3] O. Aguilar, R. Tapia, A. Valderrabano and H. Minor. "Design and performance comparison of PI and adaptive current controllers for a WECS". IEEE Latin American Transactions. Vol. $13 \mathrm{~N}^{\circ}$ 5, pp. 1361-1368. 2015. ISSN: 1548-0992. DOI: 10.1109/TLA.2015.7111990.

[4] Y.K. Wong and A. B. Rad. "Comparision of performance of a fuzzy controller for a heating a PID”. Microprocessors and Microsystems. Vol. $18 \mathrm{~N}^{\circ}$ 7, pp. 401-407. 1994. ISSN: 01419331. DOI: 10.1016/0141-9331(94)90068-X.

[5] K. Ogata. "Ingeniería de control moderno". Prentice Hall. 5th edición, pp. 1-60. Madrid, España. ISBN: 978-84-8322-660-5. 2003.

[6] S.H.H. Haque, H. M. I. Hassan and S. M. A. Hossain. "Comparison of control system using PLC \& PID”. ASEE 2014 Zone I Conference. University of Bridgeport. Bridgpeort, CT, USA. 2014.

[7] L. Guo. "Design projects in a programmable logic controller (PLC) course in electrical engineering technology". Technology
Interface Journal/Fall. Vol. $10 \mathrm{~N}^{\mathrm{o}} 1.2009$. ISSN: 1523-9926.

[8] F. Moreno, J. Mondragón and J. Becerra. "Implementations the PID algorithm in the programmable logic controller under industrial communications network". Scientia et Technica. Año XVII. No 50, pp. 33-39. 2012. ISSN: 0122-1701.

[9] C.D. Johnson. "Process control instrumentation technology". Pearson. 8th edition. London, UK. ISBN: 978-0131194571. 2006.

[10] C. Saygin and F. Kahraman. "A Web-based programmable logic controller laboratory for manufacturing engineering education". The International Journal of Advanced Manufacturing Technology. Vol. $24 \mathrm{~N}^{\mathrm{o}}$ 7-8, pp. 590-598. 2004. ISSN: 0268-3768. DOI: 10.1007/s00170-003-1787-7.

[11] D.A. Tibaduiza and I. Amaya. "Implementación de un control fuzzy para el control cinemático directo en un robot manipulador". Ingeniare. Revista chilena de ingeniería. Vol. $19 \mathrm{~N}^{\circ} 3$, pp. 312-322. 2011. ISSN: 0718-3305. DOI: 10.4067/S0718-33052011000300002.

[12] S. Chitra and V. Raghavan. "Conveyor control using programmable logic controller". International Journal of Advancements in Research \& Technology. Vol. $3 \mathrm{~N}^{\circ} 8$, pp. 25-31. 2014. ISSN: 2278-7763.

[13] I. Ruge. "Método básico para implementar un controlador digital PID en un microcontrolador PIC para desarrollo de aplicaciones a bajo costo". Aplicaciones en Control y Potencia en la Industria. Universidad de Cundinamarca. Grupo de Investigación en Tecnologías de la Información y las Comunicaciones. Bogotá, Colombia. 2008.

[14] J.E. Noriega. "Diseño e implementación de un controlador PID digital en un microcontrolador utilizando técnicas de prototipado rápido". Tesis de grado. Universidad Pontificia Bolivariana. Bucaramanga, Colombia. 2011.

[15] A. Maghsadhagh. "Implementation of PID controller by microcontroller of PIC (18 Series) and controlling the height of liquid in sources". Advances in Robotics \& Automation. Vol. $5 \mathrm{~N}^{\circ}$ 3, pp. 3-6. 2016. ISSN: 2168-9695. DOI: $10.4172 / 2168-9695.1000156$.

[16] S. Herrera-Aristizábal, J. Hincapié-Correa, L. Ríos-González and S. López. "PIDfuzzy of DC motors using Raspberry PI". 
Visión Electrónica. Vol. 14 No 1, pp. 1-30. 2020. ISSN: 1909-9746. DOI: $10.14483 /$ issn.2248-4728.

[17] M. Boutouba, A. El Ougli, S. Miqoi and B. Tidhaf. "Design and experimentation of a control system implemented on Raspberry Pi 3 board for photovoltaic systems using SEPIC converter". Journal of Electrical Systems. Vol. $13 \mathrm{~N}^{\circ}$ 4, pp. 661-677. 2017. ISSN: 1112-5209.

[18] Y. Ramchandra, M. Salim, T. Ankush and P. Vasantrao. "Self-balancing robot using Raspberry Pi and PID controller". International Journal of Innovative Science and Research Technology. Vol. $6 \mathrm{~N}^{\circ} 4$, pp. 321-322. 2021. ISSN: 2456-2165.

[19] V. Rivera and G. Arancibia. "Diseño e implementación de un observatorio robótico teleoperado basado en robot operating system". Ingeniare. Revista chilena de ingeniería. Vol. 26, número especial, pp. 12-19. 2018. ISSN: 0718-3305. DOI: 10.4067/S0718-330520180005000 12.

[20] J.C. Basilio and S.R. Matos. "Design of PI and PID controllers with transient performance specification". IEEE Transactions on Education. Vol. $45 \mathrm{~N}^{\circ}$ 4, pp. 364-370. 2002. ISSN: $1557-$ 9638. DOI: 10.1109/TE.2002.804399.

[21] I.A.R. Ruge. "Optimización de señal de control en reguladores PID con arquitectura anti-reset Wind-Up". Tecnura. Vol. $15 \mathrm{~N}^{\circ} 30$, pp. 24-31. 2011. ISSN: 2248-7638. DOI: 10.14483/ udistrital.jour.tecnura.2011.2.a02

[22] W. Gutiérrez, M. Fernández and W. Mantilla. "The joint training, a SENA learning model for latin america". IEEE Latin America Transactions. Vol. $14 \mathrm{~N}^{\mathrm{o}}$ 6, pp. 2997-3002. 2016. ISSN: 1548-0992. DOI: $10.1109 /$ TLA.2016.7555287.

[23] Siemens. "SIMATIC S7-1200 programmable controller". 4th Edition, pp. 2-119. Nürnberg, Germany. 2012.

[24] Siemens. "SIMATIC S7-1200, S7-1500. Regulación PID manual de funciones". Siemens AG, pp. 94. Nürnberg, Germany. 2018.

[25] K. Ogata. "Sistemas de control en tiempo discreto". Prentice Hall Hispanoamérica. 2da edición, pp. 23-172. Ciudad de México, México. ISBN: 968-880-539-4. 1996. 